\title{
Educating Leaders 2018: Abstracts From AACOM's Annual Conference
}

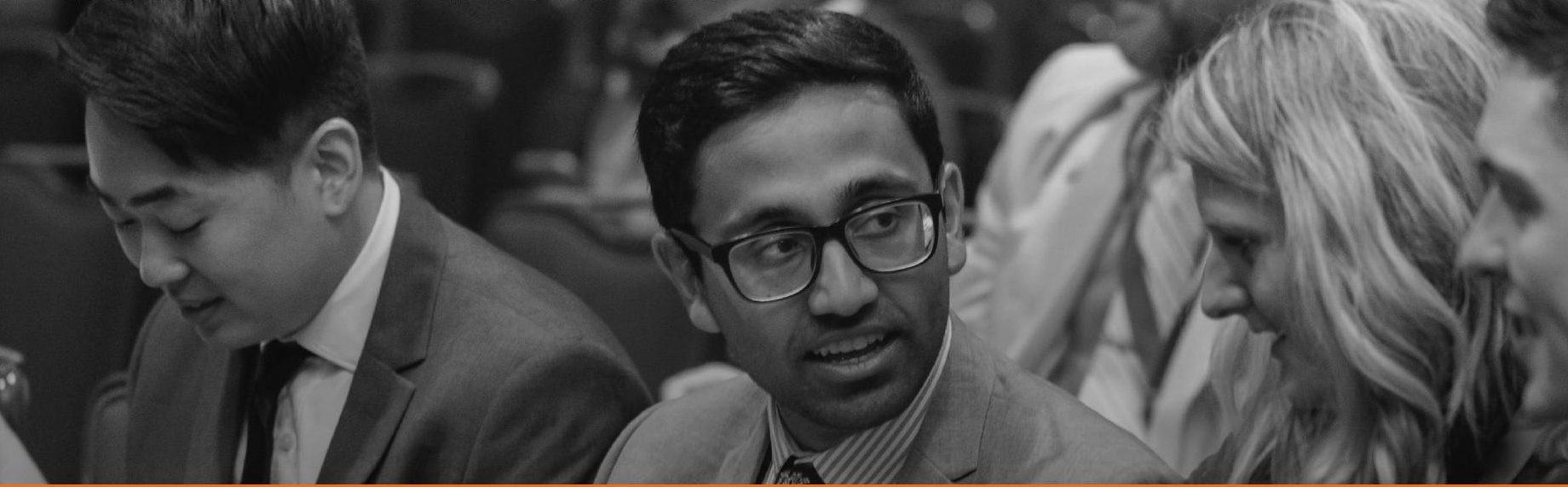

Educating Leaders is the annual conference of the American Association of Colleges of Osteopathic Medicine (AACOM). In preparation for this event, the Call for Presentations receives hundreds of high-quality abstract submissions. Presentation submissions for Educating Leaders 2018 were based on seven focus areas: Advocacy and Policy, Graduate Medical Education, Humanism and Mentoring, Learner Affairs, Osteopathic Medical Education, Professional Development, and Research Support and Technology. Following Educating Leaders 2018, session presenters were provided the opportunity to submit their sessions for publication. These sessions were reviewed by fellows (listed below) of the National Academy of Osteopathic Medical Educators-we thank them for their service. The following abstracts comprise the research and scholarly work of faculty, administrators, students, and physicians from across the osteopathic medical education continuum and were accepted by AACOM and the American Osteopathic Association for publication in The Journal of the American Osteopathic Association. (doi: 10.7556/jaoa.2018.126)

\section{Reviewers}

Mark Andrews, PhD

Lake Erie College of Osteopathic Medicine

Timothy Barreiro, DO, MPH

Ohio University Heritage College of Osteopathic Medicine

Neil Chamberlain, PhD

A.T. Still University, Kirksville College of Osteopathic Medicine

Mark Hernandez, MS, PhD

Alabama College of Osteopathic Medicine

Sherry Jimenez, EdD

Lincoln Memorial University - DeBusk College of Osteopathic Medicine

Christopher Keller, PhD

Lake Erie College of Osteopathic Medicine

Michael Kuchera, DO

Marian University College of Osteopathic Medicine

Harold Lausen, DO, MA

Southern Illinois University School of Medicine

Drew Lewis, DO

Des Moines University College of Osteopathic Medicine

Machelle Linsenmeyer, EdD

West Virginia School of Osteopathic Medicine

Elizabeth McClain, PhD

William Carey University College of Osteopathic Medicine

\section{Luke Mortensen, PhD}

Idaho College of Osteopathic Medicine

Evelyn Schwalenberg, DO, MS

University of New England College of Osteopathic Medicine Patricia Sexton, DHEd

American Association of Colleges of Osteopathic Medicine Mark Speicher, PhD

American Association of Colleges of Osteopathic Medicine

Other

Stephen Shannon, DO, MPH

American Association of Colleges of Osteopathic Medicine

Mark Speicher, PhD

American Association of Colleges of Osteopathic Medicine

Lynn Bragan

American Association of Colleges of Osteopathic Medicine Erin Ross

American Association of Colleges of Osteopathic Medicine

Lindsey Jurd

American Association of Colleges of Osteopathic Medicine

Lucy Nye

CTI Meeting Technology 
TISSUE: A Prematriculation Program Developed by Current Medical Students for Incoming Medical Students

Kathleen Ackert; Stephanie Michalik; Kristin Oller; Brandon G. Twombly; Mark M. Ujevich;

Marina D'Angelo

Philadelphia College of Osteopathic Medicine (PCOM)

Context: The TISSUE (Teaching Introductory Study Skills Utilizing Experience) program was created to provide exposure to the anatomical sciences for students who had not taken courses in anatomy and/or histology, had not received an undergraduate degree in a science concentration, and/or who had not been recently involved in an academic setting. TISSUE was developed for incoming medical students by current medical students.

Objective: The main goal of TISSUE was to mitigate the perceived academic, emotional, and personal stress of incoming first-year medical students. The program included academic skills, study strategies, time management, and student wellness. TISSUE was offered during July 2017 to the incoming PCOM class of 2021 students (OMS I) with the option to participate in an in-person or online cohort.

Methods: The TISSUE program was created to provide exposure to the anatomical sciences in a low-stakes environment. In April 2017, TISSUE coordinators sent a letter to the entire incoming class of 2021 inviting them to take part in the pilot year of the program. It was emphasized that the program would most benefit students who pursued nonscience majors at the undergraduate level, nontraditional students, students pursuing medicine as a second career, and students who had not previously taken anatomy and/or histology. Based on previous research regarding student-led initiatives, it was decided that the entire course would be completely student run and focus on learning outcomes as opposed to learning objectives. Ninety-seven rising OMS I students participated-31 in person and 66 online.

Results: A series of surveys on "perceived stress" were administered to the class of 2021 (OMS I). These data are in the process of being stratified to compare self-reported stress levels between those who participated in TISSUE, those who received an MS in biomedical sciences from PCOM, and those who did not have any prematriculation association with PCOM. This research recently received a grant from the American Osteopathic Association (AOA). The following student response was typical: "This class helped me feel more prepared for medical school. I loved how the TISSUE program emphasized good habits of a successful medical student so that we'll be less likely to fail something, panic, then be forced to figure things out on our own. The program offered a solid approach to year 1. I also loved that the program included information on how to balance other areas of life with school."

Conclusion: The TISSUE program was wellreceived by the rising OMS I students. Each learning outcome was fulfilled. As reflected enthusiastically by students in an anonymous postclass survey, the students enjoyed the class as a whole, became comfortable living in a new city, and were thankful for their early exposure to cadaveric anatomy. Preliminary studies by the PCOM Biomedical Sciences Department show that the students who participated in TISSUE had better grades in the Structural Principles of Osteopathic Medicine course compared with other students. Four new facilitators were selected from the class of 2021 after an interview process, and TISSUE 2018 is slated to begin in July. 


\section{Clinical Mentoring at the PCOM Philadelphia Campus: A Student-Originated Program \\ Michael A. Becker; Emily J. Eshleman; Danielle \\ C. Estrada; Patrick Lannutti \\ Philadelphia College of Osteopathic Medicine (PCOM)}

Context: The PCOM Philadelphia campus Student Clinical Mentorship Program was initiated by medical students who saw a need for greater connection between students and faculty. Those students also recognized that much of learning how to be a physician occurs outside the classroom and by example.

Objective: This program was designed to guide students through their transition into medical school and provide a personal connection to a clinician mentor with the intent of supporting students in their professional development.

Methods: A survey was sent out to first-year osteopathic medical students to determine their initial interest in mentorship relationships with faculty osteopathic physicians. The effectiveness of the program is also monitored through term surveys sent out 3 times per year to the larger student body; changes to the program have been based on that feedback. The program design uses a family model made up of osteopathic faculty mentors and students from different class years. The family model emphasizes the personal nature of a mentor and maximizes students' well-being and professional guidance through horizontal (among students) and vertical (older students to younger students and mentor to mentee) mentorship. This model makes use of larger group family meetings, as well as 1-on-1 meetings between the clinical mentor and mentee.

Results: The initial survey received 61 responses and found that 53 students were interested in a physician mentor. Students specifically wanted someone to go to for transitioning issues (21 of 59 respondents), career advice (56 of 59), professional networking (51 of 59), and a role model for future career (47 of 59). At the end of the program's first year, 47 of 75 students indicated that the program met their expectations, and 43 of 75 students indicated that they planned to use the program for career support/networking as a second-year medical student.

Conclusion: The program was initiated by students in collaboration with faculty, and it continues to be led by 2 faculty members and a committee of students. Students serving a primary role in the leadership of the Student Clinical Mentorship Program ensure that the focus of the program remains driven by student needs. While this survey-driven program helps coordinators stay connected with the student population, the survey response rate does pose certain limits. As the program continues to grow and data can be consolidated over many terms, program coordinators anticipate being able to guide the program based on patterns. The Student Clinical Mentorship Program is now in its second year. It is expected that the embedded culture of mentorship will continue to have a positive impact on student well-being and professional development as students continue to learn what it means to be an osteopathic physician, both inside and outside the classroom.

\section{Osteopathic Educational Frontiers: Turning Old Tales Into New Stories John Casey; Vishnu V. Mudrakola; Andrew Little OhioHealth Doctors Hospital}

Context: As osteopathic medical education advances, including the use of technology and alternatively-sourced information, it is imperative that teaching methods be adapted to facilitate the simultaneous goals of content delivery and participant engagement.

Objective: The primary purpose of this pilot research was to establish that a flipped classroom 
model of education can deliver content while improving learner satisfaction with the experience.

Methods: Thirty osteopathic medical students from first- through fourth-year training were randomly assigned to receive focused subject matter training either by traditional classroom (lecture) or flipped classroom methods on the topic of seizure management. Postintervention testing to assess material retention was conducted, as well as survey of participants' preference for the educational technique they were assigned. Statistical analysis was performed using a $t$ test for independent means. The study had institutional review board approval.

Results: There were no significant differences in test scores between the traditional and flipped classroom groups $(P=.14)$. However, there was a strong and statistically significant preference demonstrated for the flipped classroom method of teaching ( $P=.018$ in terms of satisfaction and $P=.011$ in terms of feeling prepared to take their examination).

Conclusion: Although this was a small pilot research project at a single institution, when taken in totality with current educational theory, the study suggests that novel presentation methods like the flipped classroom may deliver content as effectively as traditional lecture and be far more engaging for the learner. Not specifically addressed in this study was educator preference. However, successful collaboration between educators and learners makes the process of curriculum design an active process and ensured buy-in from all participants. As the first emergency medicine residency to obtain osteopathic recognition through the single graduate medical education accreditation system, participating researchers used this method to modify the traditional classroom model and implement a novel emergency medicine curriculum. The researchers found that innovative curriculum design, coupled with multimodality educational sources such as free, open access medical education, can aid in providing a flipped educational environment enjoyed by both the learner and the educator.

\section{Situation Judgment Tests (SJT), a Fresh Approach to Medical School Admissions \\ Leanne M. Chrisman-Khawam; Samantha Baker Ohio University Heritage College of Osteopathic Medicine (OU-HCOM), Cleveland}

Context: With 40 years of validation and study, the United Kingdom and other countries use situation judgment tests (SJT) for medical school admission or residency selection criteria. Research reveals them as deliberate, validated, and superior to interview in examination of desirable professional attributes. When used in addition to traditional academic attributes, SJT scenarios help predict future performance on professional attributes. The largest drawback is the narrow variation from best to worst performance.

Objective: The objective of the study was to determine the impact on admissions of the use of SJT among applicants to OU-HCOM.

Methods: Four SJT scenarios, each with 5 to 8 response stems, were created based on validated human resources scenarios modified to fit early medical student situations that do not require specific medical knowledge. Scenarios were rated by admission stakeholders including residency faculty, program directors, admissions staff, current family medicine residents, medical school faculty, and deans. To evaluate SJT as a selection process, scenarios will be administered to accelerated family medicine pathway candidates, approximately 50 of 400 secondary applications sent annually by 1 US medical college, $12.5 \%$ of applicants considering 1 of 3 campuses in the 2017-2018 admissions cycle. 
Results: Applicants rated the appropriateness of each SJT response on a 5-point Likert scale and ranked the responses from least to most appropriate. During their interviews, applicants were queried on the perceived core value that drove their responses. They were subsequently asked to complete the Bore-Powis Personal Qualities Assessment, Emotional-Social Competency Inventory, Jefferson Empathy Scale, Groningen Reflection Ability Scale, and Tolerance for Ambiguity Scale. The researchers plan to explore associations between SJT score, SJT stated core value, personality traits, other pre-admission measures (eg, grade point average, Medical College Admission Test scores), and behavioral-based interview scores. Initially, no statistically significant differences in SJT scores between those selected by interview and those not selected were found. However, the researchers note that the study was underpowered to show statistical significance. Results from other scales are still pending.

Conclusion: SJT responses are useful tools to examine decision making and applicant core values. Additional research will be required to reach adequate power to show significance.

\section{Introducing Allopathic Medical Students to Osteopathic Principles and Manipulative Techniques \\ Christopher Frothingham ${ }^{1}$; Trent Reed ${ }^{1}$; Kyle Wieschhaus ${ }^{2}$; Joseph $\mathrm{Nye}^{2}$; Jacqueline Greene ${ }^{2}$ ${ }^{1}$ University of New England College of Osteopathic Medicine (UNECOM); ${ }^{2}$ Stritch School of Medicine at Loyola University, Chicago}

Context: As of 2020, all residency training programs will fall under a single governanceAccreditation Council for Graduate Medical Education (ACGME). Some residency programs may decide to apply for osteopathic recognition (OR). Some of those residency positions will be reserved for osteopathic medical school graduates or allopathic graduates who demonstrate some level of preparation for entry into an osteopathicfocused position. With the approaching graduate medical education (GME) merger and the paucity of data related to exposing allopathic medical students to osteopathic principles and practice, research in this area is needed.

Objectives: (1) To adequately introduce allopathic medical students to the fundamentals of osteopathic manipulative treatment (OMT) in key areas, including philosophy, structural examination basics, soft tissue and lymphatics, myofascial release, straincounterstrain, muscle energy, and HVLA. (2) To describe an elective curriculum structure to introduce allopathic medical students to osteopathic principles and practice and manipulative treatment.

Methods: Design: This prospective cohort study consisted of a 3-month elective courseIntroduction to Osteopathic Medicine-at an allopathic medical school, which consisted of viewing 8 asynchronous podcasts and participating in 5 hands-on skills sessions. Setting: The podcasts were developed by osteopathic physicians at UNECOM under the guidance of Chris Frothingham, DO, as well as the Stritch School of Medicine at Loyola University in Chicago under the guidance of Trent Reed, DO. The video podcast content was briefly reviewed prior to hands-on application of the techniques in Loyola University's simulation laboratory. Participants: Twenty-eight allopathic medical students of OMS II to OMS IV standing completed the course. Main Outcome Measures: Main tools used to assess outcome measures were before and after Likert questionnaires, multiple-choice question (MCQ) examinations, and evaluation of diagnosis and management of 2 somatic dysfunctions. Outcome measures of success included an objective increase in performance on the MCQ examination, a more positive attitude toward osteopathic medicine on the questionnaire, and passing of the final practical assessment. 
Results: Improvement in core osteopathic knowledge as objectively measured by MCQ pretests vs posttests was statistically significant $\left(\mathrm{M}_{\mathrm{diff}}=12.46\right.$ points; 95\% CI, 10.36-14.57; $P<.001)$. There was no meaningful difference in improvement between OMS II, OMS III, or OMS IV students ( $P=.14$ ). The study found that $85.8 \%$ of students agreed or strongly agreed that taking this elective reinforced previously learned anatomical knowledge. Similarly, $92.8 \%$ of students agreed or strongly agreed that after taking this elective they are more comfortable with the musculoskeletal assessment of their patients. All students successfully diagnosed and managed 2 somatic dysfunctions based on a standardized rubric. Lastly, there was a statistically significant increase in self-rated understanding of osteopathic principles $(P<.001)$ and likelihood to apply for a residency position with osteopathic-focused education in a program with osteopathic recognition $(P<.001)$.

Conclusion: Students demonstrated significant improvement in core osteopathic knowledge from MCQ pretest to posttest. Students displayed absorption of osteopathic manipulative examination and treatment as evidenced by passing their final practical examination. Students' attitudes toward osteopathic medicine have become more favorable as well as their perceptions of their own knowledge and skills.

\section{Collaboration in a Special Master's Program Increases Diversity in an Osteopathic Medical College \\ Christina Goode ${ }^{1}$; Gerald R. Thrush ${ }^{2}$; Susan E. Mackintosh ${ }^{2}$; Colleen Talbot ${ }^{3}$ \\ ${ }^{1}$ Graduate College of Biomedical Sciences, Western University; ${ }^{2}$ Western University of Health Sciences/ College of Osteopathic Medicine of the Pacific (WesternU/COMP), Pomona, California; ${ }^{3}$ WesternU/ COMP, Lebanon, Oregon}

Context: A diverse population of health care providers is important to help reduce health care dispar- ities and provide culturally sensitive care in underserved areas. The Master of Science in Medical Sciences (MSMS) program is a special master's program at Western University of Health Sciences that is designed for diverse and underrepresented students. The program can serve as a stepping stone to professional school for students who show promise but are not competitive for direct admission. Collaboration between the program and WesternU/COMP has been essential in the success of the program. This study describes the performance of the students in this program on the 2 campuses of WesternU/COMP.

Objective: To describe the best practices for developing a curriculum for a transitional master's program, and to describe the role of program graduates in advancing the social mission of the health professions institution.

Methods: All students who transitioned to WesternU/COMP from the MSMS program since its inception in 2010 were automatically included in this study ( $\mathrm{N}=123)$. In addition to recording ethnicity/race and socioeconomic status for each student, prematriculation (before the MSMS program) metrics (grade point average [GPA] and Medical College Admission Test scores), MSMS program GPA and relative performance in the preclinical curriculum, and COMLEX-USA Level 1 board scores were collected. Data were analyzed using regression analyses or $t$ tests for independent means. Additionally, information on campus involvement, community service activities, and leadership positions was also obtained (either from the office of student affairs or through direct polling of MSMS alumni). This study received approval from the Western University of Health Sciences Institutional Review Board (\#X17/IRB/079).

Results: By tracking the performance of MSMS graduates through their preclinical program, the researchers were able to obtain information that allowed data-driven changes to the MSMS 
program, including the curriculum, the MSMS admissions process, and the admissions criteria used to determine which students would be allowed to transition to WesternU/COMP. Results have indicated that the best predictor of MSMS student performance at WesternU/COMP was their MSMS program GPA, which strongly correlates with their relative class rank $\left(R^{2}=0.598\right.$, $P<.001)$ at WesternU/COMP for their preclinical coursework. Their medical school class rank, in turn, correlates with their performance on the COMLEX-USA Level $1\left(\mathrm{R}^{2}=0.528\right)$. The research also indicated that MSMS program alumni have a positive effect on their WesternU/COMP peers, as approximately $75 \%$ of all MSMS alumni at WesternU/COMP reported that they are/were involved in campus clubs and/or community service. Many have also served in a variety of leadership roles, such as the student government (including as class president) and the WesternU/ COMP Council of Leaders. Additionally, MSMS graduates helped re-establish campus chapters for national organizations that enhance diversity and inclusion, often holding key leadership positions.

Conclusion: This study provides evidence that data-driven postbaccalaureate programs such as the MSMS program can prepare students for professional school before matriculation and make a significant contribution to diversifying health professions schools after matriculation, ultimately leading to a more diverse and culturally-humble workforce.

\section{The Healing Path: A Student Initiative to Address Mental Health \\ Samantha Gottlieb; Alexander Nello; Matthew Goldfinger \\ New York Institute of Technology College of Osteopathic Medicine (NYITCOM)}

Context: Students and practitioners in the health care field are often focused on the ultimate goal to heal others but forget the importance of caring for themselves. In 2016, a meta-analysis ${ }^{1}$ reported that the prevalence of depression among medical students was $27.2 \%$, with other studies reporting that as many as $60 \%$ of students meet the criteria for diagnosis of major depressive disorder during their preclinical studies. Burnout was reported in about $50 \%$ of students during their training. ${ }^{2}$ Common to all medical students is their lack of exposure to the outdoors - always studying or training inside under harsh fluorescent lights, monitors, and buzzers. There is robust support for the importance of nature relatedness and its impact on happiness and self-assessment of wellbeing. ${ }^{3,4}$ Exposure to nature can also prevent burnout and reduce anxiety. ${ }^{4}$

Objectives: "The Healing Path" hoped to address these issues by providing an outdoor refuge where students could relax, reflect, and reconnect with their goals and values. The aims of this project included facilitating personal well-being and supporting the development of students into competent and compassionate healers.

Methods: This project started as a student initiative. Faculty then solicited input from both NYITCOM and the NYIT School of Health Professions and incorporated the diverse visions into a cohesive project.

Results: The Path, which surrounds a pond in the heart of our campus, is composed of 8 reflecting areas each portraying a different tenet that is essential to a healthy and effective career in medicine. Each space contains a weatherproof notebook to allow those who are reflecting to share their thoughts and read the entries of others. The goal was to not only stimulate introspective writing, but to provide a forum for students to learn from past student experiences written in the books. As the books are filled, they will be added to the medical library as a permanent installation for future students to utilize. Finally, The Path 
also features artwork created by students to further promote wellness by art appreciation.

Conclusion: The project was created to be a dynamic installation, ever-changing to adapt to the needs of future students, faculty, and staff.

\section{References}

1. Rotenstein LS, Ramos MA, Torre M, et al. Prevalence of depression, depressive symptoms, and suicidal ideation among medical students. JAMA. 2016;316(21):2214-2236.

2. Dyrbye LN, Thomas MR, Massie FS, et al. Burnout and suicidal ideation among U.S. medical students. Ann Intern Med. 2008;149(5):334-341

3. Capaldi CA, Dopko RL, Zelenski JM. The relationship between nature connectedness and happiness: a meta-analysis. Front Psychol. 2014;5:976.

4. Martyn $\mathrm{P}$, Brymer E. The relationship between nature relatedness and anxiety. J Health Psychol. 2016;21 (7):1436-1445

\section{An Orientation Standardized Patient Experience to Promote Critical Self-Reflection}

Amber J. Heck; Carol Browne; Linda Grace Solis University of the Incarnate Word School of Osteopathic Medicine (UIWSOM)

Context: Standardized patients (SPs) are used in medical education to provide a realistic learning resource for the development of clinical skills; however, SPs are underused as a resource to address the nonclinical skills essential to success in medical education and practice.

Objective: We hypothesized that an SP experience, as part of an objective structured clinical examination (OSCE) implemented during orientation, can be designed to promote self-reflective practice.

Methods: This collaborative effort was accomplished through the contributions of multidisciplinary faculty and student affairs staff convened within a program development committee. The intervention was designed using the ADDIE (analyze, design, develop, implement, evaluate) framework model, and the study was carried out using action research design. Participants were all first-year osteopathic medical students $(\mathrm{N}=159)$ matriculating in fall 2017. Intervention and data collection took place during the UIWSOM orientation program using the UIWSOM standardized patient clinic and all associated resources and staff. Participants were provided a self-study document, which included assigned reading on patientphysician communication and self-assessment, and the interpersonal skills evaluation (IPSE) rubric used for scoring approximately 1 month prior to matriculation. During orientation, participants reported to the standardized patient clinic and participated in a SP encounter that was 5 minutes in length followed by a 2-minute self-evaluation and SP evaluation. The encounter was designed according to a 1-station OSCE format, and the case was developed by clinical faculty to eliminate any requirement for physical examination skills. The participants were scored by the SP using the IPSE rubric, which contains the following constructs, each with 1 to 3 questions addressing interpersonal skill demonstration: opens discussion, gathers information, establishes rapport, acknowledges patient, provides closure, and maintains professionalism. They then completed 2 postencounter selfevaluations using the same IPSE rubric: 1 upon leaving the room, and again upon completion of detailed debriefing with good and bad interpersonal skills video examples and SP feedback, led by clinical and medical humanities faculty.

Results: Post-debrief self-evaluation mean IPSE scores were statistically significantly lower than means scores by SPs $(P<.0001)$ and mean selfevaluation scores after the encounter $(P<.0001)$ according to paired $t$ test. Further, post-debrief self-evaluation scores dropped significantly within several IPSE categories according to paired $t$ test, including introducing themselves, setting an agenda, using appropriate questions, demonstrating good facilitative behavior, 
appearing empathetic $(P<.0001)$, summarizing the encounter $(P<.001)$, and appearing confident $(P<.002)$. Student evaluation of the experience was positive, with $68 \%$ of respondents $(n=41)$ identifying it as "extremely useful," the highest of 5 Likert scale options.

Conclusion: In conclusion, the orientation SP experience supported increasingly critical selfassessment, as evidenced by statistically lower student self-evaluation scores after faculty-led debrief. It did not, however, facilitate accurate self-evaluation, as evidenced by significantly lower self-evaluation scores when compared with SP scores. Future directions include investigation into and predictive validity of this exercise and its effectiveness in increasing interpersonal skills performance during structured clinical examinations in the preclinical years.

\section{Enhancing Student Metacognitive Awareness Through a Hybrid Orientation Program \\ Amber J. Heck; Kevin E. Kalinowski; Sophia Pina University of the Incarnate Word School of Osteopathic Medicine (UIWSOM)}

Context: Orientation programs are a longstanding tradition in medical education and most describe goals of improving student academic performance. The current orientation approach, which focuses on awareness of academic programs and institutional resources, may be ineffective at improving student academic performance. Ample evidence suggests that increasing metacognitive awareness, or the knowledge of one's own cognitive skills and abilities, can improve academic performance.

Objective: Participants set out to design an orientation program that supports metacognitive awareness accomplished through the collaboration of multidisciplinary faculty and student affairs staff.
Methods: The orientation program was designed in the format of the osteopathic medical school curriculum to support self-directed, case-based, and active learning in both large and small groups. Intervention and data collection took place during the UIWSOM orientation program using the academic facilities and equipment at the UIWSOM Brooks Campus. Academic resources used by study participants did not exceed that which was already available through the UIWSOM library. Study personnel included UIWSOM faculty and staff. Participants were all first-year osteopathic medical students $(\mathrm{N}=159)$ matriculating in fall 2017. This study was approved by the UIW Human Subjects Institutional Review Board. Participants were provided with an orientation learner guide 1 month in advance. The guide included the program description and goals, instructions to access the resources available, and descriptions of the methods of instruction to be employed. For each session, the guide provided a title, outcomes that students are expected to gain by the end of the session, objectives that students are expected to prepare ahead of time, and suggested readings. During orientation week, participants attended 26 hours of sessions over 5 consecutive days, and sessions were delivered in the format of the UIWSOM curriculum, which included: small group casebased learning, large group active learning sessions, active learning laboratories, and a standardized patient experience. Content addressed the academic program overview, institutional resources, mindset, metacognition, professional identity formation, patient-physician relationship, and clinical reasoning. Participants were surveyed before and after implementation using the validated instrument Metacognitive Awareness Inventory (MAI).

Results: The increase in MAI total scores after intervention was not statistically significant. However, analysis of instrument constructs (declarative, procedural, and conditional 
knowledge) revealed a statistically significant increase in declarative knowledge after intervention $(P<.0001)$ according to paired $t$ test. Declarative knowledge is the factual knowledge of one's skills, intellectual resources, and abilities as a learner. Student evaluation of the experience was positive, with $93 \%$ of respondents $(n=45)$ agreeing that the format of orientation helped prepare them for the UIWSOM curriculum.

Conclusion: The data collected support the conclusion that an orientation program can be designed to increase metacognitive awareness, a skill set long known to positively impact academic performance. Alternatively, the orientation experience is proof of concept that the active learning modalities used in the academic curriculum can be applied to address matters of student success in the co-curriculum.

\section{Finding HOME: Humanism in Osteopathic Medical Education}

Tami Hendriksz

Touro University College of Osteopathic Medicine-CA

Context: The rigors of current medical education often strip learners of their humanity, resulting in decreased empathy, increased rates of burnout, and high rates of depression and suicide. Osteopathic physicians are trained on the importance of individualized patient care. Osteopathic medical educators teach students to see each patient for the unique human being that they are; to consider every patient's mind, body, and spirit; and to incorporate those factors in their ideal path to good health. However, medical educators rarely have the time or personnel to model this individualized treatment to students. Too often, learners with different learning styles are lumped into large classes and expected to follow a single path to the same goal.

Objectives: It is important to learn more about each individual patient, discover the underlying cause of the symptom in each patient, and prescribe an individualized treatment plan. However, often with medical student and physician burnout, physicians prescribe the same treatment to everyone without taking the first step to identify the underlying cause and see individuals for who and what they are.

Method: Humanism in Osteopathic Medical Education (HOME) is a distinct program at Touro University College of Osteopathic Medicine-CA with the goal to promote students' humanity, sense of community, and support. This program collaborates with learners, allowing them to help prioritize what issues need to be addressed and the best means in which to do so. One of the studentdriven HOME initiatives was an online forum where students can connect with one another and seek support and advice as they share some of their struggles, experiences, and stories. Students also have in-person "meet-ups," during which they get together to discuss topics such as imposter syndrome, community connection, and mental health. Students have also developed and published HOME newsletters and held a "Living with Illness" art show and performance highlighting creative expressions of those who live with or help people with illnesses.

Results: The main outcomes of HOME can be measured through the involvement and level of engagement of the student body. The variety of outlets that HOME has allows individual students to participate and connect in the ways in which they feel the most comfortable.

Conclusion: Osteopathic medical educators need to start treating themselves and their students the way that they treat patients-osteopathically. Once that is done well, it is projected that a reversal of the burnout and empathy loss that currently prevails in medical training and practice will start to take place. 


\section{Medical Education Debt and Disparities: A Comparison of DO and MD Students \\ Blythe Jonas \\ Ohio University Heritage College of Osteopathic Medicine}

Context: Student loan debt is a growing concern for all stakeholders in higher education. Understanding trends in medical student debt allows medical educators to have a greater comprehension of the current landscape of debt for osteopathic medical students.

Objective: To examine data regarding debt levels of DO and MD students, the factors contributing to reported differences in debt levels between DO and MD students, and the consequences of the differences in those debt levels for both types of medical schools and future physicians.

Methods: This analysis compared statistics regarding debt and tuition as reported by the American Association of Colleges of Osteopathic Medicine and the Association of American Medical Colleges in their annual reports from 2014 to present. In addition to conducting a comparison for all students, figures for public and private institutions were isolated and compared to determine differences among the same type of institution.

Results: In 2014, the average medical school debt for DO students was $25 \%$ higher than the mean total medical school debt for MD students in 2014. In 2015 and 2016, the mean total medical school debt for DO students was $27 \%$ higher than that of MD students. In 2017, the disparity rose to $29 \%$, with the average DO student carrying $\$ 56,524$ more debt than the average MD student. When comparing data for DO and MD students who attend the same type of institution (public or private), in every comparison, the DO students carried more debt relative to the tuition. In some cases, the debt levels were higher even though the DO granting institution mean tuition costs were less than MD institutions' mean tuition. Scholarships and grants may be one of the factors accounting for the significant difference between the debt levels of DO and MD students. In 2017, $42 \%$ of DO students received scholarships and grants, while $63 \%$ of MD students received scholarships and grants. The median amount of scholarships and grants received by DO students was $\$ 10,000$, while MD students received a median amount of $\$ 24,000$. This study also found a difference between DO and MD students in the proportion of students that have taken loans for their undergraduate education. In 2017, $50 \%$ of DO students reported having undergraduate debt, while $35 \%$ of MD students reported carrying undergraduate debt. The difference in the number of students receiving financial aid as undergraduates is likely to indicate a difference in socioeconomic status between DO and MD students, which may be another factor affecting the disparities in medical education borrowing and debt.

Conclusion: More research must be done to have a clear picture of the drivers of debt for osteopathic students, and the implications of such debt. Osteopathic medical schools need to remain conscious of these disparities and be proactive in implementing approaches to prevent, address, and mitigate the inequities in the debt levels of their students.

\section{Recent Progress Through Interactive Blended Learning in Cardiovascular Medicine \\ Jason E. Kaplan; Erik Langenau \\ Philadelphia College of Osteopathic Medicine}

Context: Within the field of cardiovascular medicine, it is a common difficulty to keep students 
engaged while reviewing evidence-based medicine in a meaningful way in the online learning environment.

Objective: This program is designed to review strategies to develop an online blended learning program for osteopathic medical students in the field of clinical cardiology. The program is intended to help preceptors better interact with their students and communicate the necessary variety of information, cases, and evidence-based studies. Further, it will describe how to implement these changes in a coherent manner that allows for uniform education at nearly 50 clinical training sites while preserving individualized learning.

Methods: This program describes the manner that was used to refine the technologies to engage students, focus on required online assignments, and provide an abundance of resources from which students can learn. This program was implemented with 280 students at 30 clinical training sites over 18 months. This has been an effective supplement to their in-house/face-to-face cardiovascular education in both the inpatient and the outpatient settings. Researchers describe challenges of online, blended learning in cardiology, including: (1) the need to optimize student workload to facilitate between online learning and face-to-face education; (2) setting appropriate expectations of online learning to supplement in-house academic exposure; (3) developing faculty support of blended learning at clerkship training sites; and (4) organizing materials into a logical manner to facilitate access for student convenience.

Results: Third-year medical students across geographically distributed clinical training sites engage in both face-to-face and online educational activities. These educational activities include online interactive discussion forums, evidence-based journal review, prerecorded didactic lectures, and interactive online learning practice tools. The challenges of online blended learning have been addressed through (1) a strong integration of interactive workload with the objectives of face-to-face learning; (2) creating goals that keep students engaged both online and at their clerkship sites; (3) providing online access and resources to site directors; and (4) using a central course management system to establish a standardized curriculum over the 30 clinical training sites.

Conclusion: The establishment of an online blended learning environment for third-year osteopathic medical students is a necessary supplement to the face-to-face clinical education in today's educational climate. By reviewing multiple programs and educational activities, it is possible to enable online preceptors to implement interactive learning of evidence-based cardiovascular medicine.

\section{Developing Interprofessional Leadership in Evolving Models of Practice and Education \\ Janice A. Knebl ${ }^{1}$; Jennifer Jurado Severance ${ }^{1}$; Anita Chopra²; Elyse Perweiler ${ }^{2}$ \\ ${ }^{1}$ University of North Texas Health Science Center at Fort Worth Texas College of Osteopathic Medicine; ${ }^{2}$ Rowan University School of Osteopathic Medicine}

Context: There are 44 Geriatric Workforce Enhancement Programs (GWEP) funded by the Health Resources and Services Administration (HRSA) to integrate geriatrics education into primary care training, increase patient and family engagement, and improve health outcomes. With the increasing focus on value-based and patientcentered health care, there is demand for health care professionals to work effectively in teams and lead change within their organizations. Practicing health professionals want and need assistance in the implementation and continuous improvement of geriatrics care in the primary care setting. 
Objective: The objective of this study is to review the development and impact of 2 GWEP models focused on interprofessional approaches to leadership in geriatrics care.

Methods: The University of North Texas Health Science Center's GWEP partnered with a private university to develop a 10-month Geriatric Practice Leadership Institute designed to empower interprofessional teams and teach them leadership, quality improvement skills, knowledge of aging services, and population health. The curriculum was developed with the aim of enhancing each participant's understanding of personal leadership skills and how to create and participate in effective interprofessional teams that provide value-based, patient-focused care to older adults in the developing primary health care systems. Teams created meaningful interventions designed to improve quality geriatrics care and patient safety. Rowan University's GWEP developed a geriatrics primary care infusion model for treatment of high-risk, high-cost, complex patients in postacute and ambulatory care settings. Using systematic case review led by a geriatrician, and with input from an interprofessional team, recommendations were made to the primary care physician. The goal was to reduce length of stay in postacute care, decrease readmissions, reduce costs, and prepare the patient and caregiver for discharge. In the ambulatory setting, systematic case reviews of high-cost, complex patients focus on decreasing hospitalizations and linking patients to appropriate community-based resources.

Results: Fifty-two health care professionals in 12 interdisciplinary teams participated in the Geriatric Practice Leadership Institute in 2016-2018 and 2017-2018. Leadership training for interprofessional teams resulted in increased provider knowledge and confidence in care management of geriatric clients and improved outcomes for older adults and caregivers. Preliminary data showed that the geriatrician-led geriatrics infusion model demonstrated increased geriatric assessments, reduced health care utilization, and resulted in cost savings and improved quality of care.

Conclusion: Training programs for interprofessional teams in leadership can enhance interprofessional skills and improve geriatrics care in primary care settings. These models engage crosssector stakeholders in leadership development to address gaps in care at individual, system, community, and population levels.

\section{Portfolios, Mentoring, and How MBTI Preferences Relate to Residency Specialty Choices}

Patricia K. Krohmer; Lisa Szymanski; Tina Marino; Emily Whitis

Midwestern University Chicago College of Osteopathic Medicine (MWU/CCOM)

Context: The Costin Institute's mission is to help osteopathic physicians become better educators, leaders, and administrators. To achieve that mission, the Costin Institute provides opportunities to develop knowledge, skills, and abilities for each participant's focus. Recently, the Costin Institute initiated 2 programs: The Portfolio Project and The Mentoring Project, both of which will be closely monitored and reported on as they develop. The relevance of educational research is reinforced and encouraged. One example of research being conducted by Costin Institute scholars is a study designed to compare the Myers Briggs Type Indicator (MBTI), which measures the personality preferences of osteopathic medical students to their choice of residency specialty. The research results may not only assist students who are undecided in their residency choice, but could also help them understand themselves better, 
understand others, and embrace diversity. Students can include this information in an ePortfolio, which would demonstrate self-awareness. Additionally, faculty members involved in mentoring students regarding career choices could benefit from the conclusions of the study. The MBTI has been used to study variations of normal personality types and has, in the past, been compared with individual student performance on various standardized examinations. In addition, studies have been conducted with allopathic students and their residency choices. To date, no study has compared osteopathic medical students' MBTI type with their residency specialty of choice.

Objective: To determine the association between the MBTI personality type of MWU/CCOM osteopathic medical students and their specialty career choice when matching for residency in the fourth year of medical school.

Methods: Data from the class of 2014 were collected as part of a pilot study. All medical students in the class of 2015 were offered to voluntarily take the MBTI assessment (in person) before beginning their fourth year of medical school and report their scores to the investigators. The assessment was administered during the integrated clinical activities (ICA) course, which was designed to assist fourth-year medical students with residency choices and/or applications. Following both the National Matching Service (NMS) and National Resident Matching Program (NRMP) matches in spring, a correlation was determined, grouping specialty type with various MBTI categories. This was done anonymously with the students' identities removed from the data analysis. The data were compared to the students' specialty choices when they entered medical school, program rankings, and their actual placement results.

Results: Emergency medicine showed preferences in 2014 and 2015 for extroversion, sensing, thinking, and perceiving. Family medicine showed preferences in 2014 and 2015 for extroversion, sensing, feeling, and judging. Internal medicine showed preferences in 2014 for extroversion, sensing, feeling, and judging. In 2015, the preferences were for introversion, sensing, thinking, and perceiving. Pediatrics showed preferences in both 2014 and 2015 for extroversion, sensing, feeling, and judging.

Conclusion: The MBTI gives the students a useful tool in determining their best residency options.

\section{The R.A.I.S.E. Program: A Summer Immersion for HS Students Promoting Osteopathic Understanding and \\ Careers \\ Kathryn Lambert; Matthew Tribble; Paula Watkins; Venkateswar Venkataraman \\ Rowan University School of Osteopathic Medicine (RowanSOM)}

Context: RowanSOM recognizes the need for innovative admissions strategies to recruit a wellqualified and diverse pool of applicants.

Objective: Rowan University created the RAISE (Recognizing Achievement and Inspiring Student Excellence) program to address this need. The RAISE program sought to create a unique, interactive pipeline program that would increase osteopathic understanding in the community while effectively recruiting high achieving students.

Methods/Program Description: The RAISE program consisted of a 5 -week summer immersion for high-achieving high school students providing an awareness of medical school education, residency training, and physician practice. Students with at least a 3.5 grade point average nominated by their guidance counselors submitted program applications and a personal statement, which helped ensure that the 24 chosen students were committed to an intensive, team-based summer 
program. Diversity and financial need were also considered; however, the program was free and included a stethoscope, blood pressure cuff, scrubs and suturing supplies for practice at home. Participants received mentorship from faculty, medical students and undergraduate students in the Rowan University combined BS/DO program. This "mini-medical school" exposure consisted of an integrated curriculum of problem-based learning cases, basic science/clinical lectures and hands-on, clinically-relevant learning, including intubation, suturing, ultrasonography laboratories, and dissection. The distinctiveness of osteopathic medicine, misconceptions surrounding osteopathic physicians (ie, DOs), and the training/career opportunities for DOs were integrated throughout the program. Students received training in obtaining a patient history, vital signs, and using a stethoscope, which were practiced on simulators and in a standardized patient laboratory. Students presented selected medical topics as poster and case presentations. Students were provided shadowing at multiple primary care locations. The curriculum also included HIPAA training, BLS Certification, and personal enrichment courses for understanding time management, learning styles, mental health concerns, wellness, and diversity. Students were surveyed weekly. The surveys were anonymous and completed through Blackboard, Rowan University's online learning platform. Students were told the surveys were not mandatory, but the lowest completion rate in any week was $70 \%$.

Results: Students who wanted medicine/health care as a career choice increased from $66.6 \%$ (week 1) to $100 \%$ (week 5). Students who said they would attend a DO school increased from $6 \%$ (week 1) to $85 \%$ (week 5), with respondents who said they had a good idea of osteopathic principles increasing from 5\% (week 1) to 77\% (week 5). Students were also asked multiple Likert scale questions about the program. Their responses showed that the participants heavily agreed they had improved their confidence in problem-solving and their ability to work in teams in what they perceived as a noncompetitive environment. Osteopathic manipulative medicine was one of the highest-rated experiences. Of the 22 senior RAISE students, 4 applied to the Rowan BS/DO program (18\%), and $100 \%$ were accepted.

Conclusion: The program saw significant increases in osteopathic understanding, personal enrichment, and interest to enter a DO program compared with preprogram results. The RAISE program proved to be not only an effective pipeline for our BS/DO program, but successful in promoting osteopathic awareness and careers in osteopathic medicine.

\section{The Remediation of Professionalism and Interpersonal Communication Skills in Residents and Students Jeffrey J. LeBoeuf Lincoln Memorial University DeBusk College of Osteopathic Medicine}

Context: The investigator sought to provide medical school faculty and student performance assessment committees with tools to remediate students and residents struggling with issues of professionalism and interpersonal communication skills (ICS)

Objectives: To identify best practices on remediation of professionalism and ICS in medical students and residents, discuss generational differences as related to professional responsibilities, explore methods of assessing professionalism, discuss a sample case study, and create remediation plans.

Methods: The investigator sought best practices for remediation of professionalism and ICS in medical students and residents through a 
systematic review of the literature. The following questions guided the search: Is professionalism a fixed character trait, or can it be taught? Can professionalism be measured? Is professionalism about values, ethics, or behavior? What do students know about professionalism?

Results: Papadakis et al (2005) found that medical students exhibiting unprofessional behavior were 3 times more likely to face discipline, later in their careers, by medical licensure boards than their peers. Those students demonstrating severe irresponsibility were 8.5 times more likely to face medical licensure board discipline. Kirk (2007) described the hidden curriculum, which is the subconscious unprofessional modeling by faculty and staff in his article "Professionalism in Medicine: Definitions and Considerations for Teaching." Hafferty's (2002) research revealed that medical students do not know "a great deal" about professionalism. Several researchers concluded that professionalism can and should be taught to medical students and residents. Holmboe et al (2010) wrote, "a competency-based approach to medical education relies on continuous, comprehensive, and elaborate assessment and feedback systems." However, few clinical preceptors or residency program faculty have a background in assessment and conduct only superficial end of rotation evaluations. Dath and Iobst (2010) called for "new strategies for authentic and regular assessment that is an essential aspect of competency based medical education." Weissbecker et al (2016) wrote, "Professionalism is a competency that can be shaped and developed over time; therefore, assessing behavior and growth across the educational continuum is essential.” Regan et al (2016) provided a model for remediation of medical residents struggling with interpersonal communication skills and professionalism: the Professionalism Milestone themes with suggested remediation strategies and Interpersonal and Communication Milestone themes with suggested remediation strategies. This article provided excellent templates for training teaching faculty and for remediating struggling students.

Conclusion: The investigator implemented best practices gleaned from the literature into a multifaceted plan to improve professionalism in faculty, staff, and students, as well to remediate problems of professionalism. The investigator developed a faculty development presentation and a fictitious case study for small group discussion of typical breaches of professionalism and ICS expectations and development of remediation plans.

\section{Ultrasound Imaging-Based Medical Education for First- and Second-Year Osteopathic Medical Students}

Inder Raj S. Makin; Chandhana Pedapati;

Kellie Huxel-Bliven; Roya Vahdatinia; Jay Crutchfield; Deborah M. Heath

A.T. Still University, School of Osteopathic Medicine in Arizona

Context: An important element of the osteopathic educational curriculum is palpatory diagnosis based on physical assessment of superficial and musculoskeletal structures. Ultrasound imaging, or ultrasonography (US), is a noninvasive, portable imaging modality that does not involve the use of radiation, which offers unique opportunities to be incorporated within the DO curriculum.

Objective: The session's objective is to describe an approach of integrating US within the osteopathic principles and practice (OPP) coursework, and expanding it to anatomy and medical skills in a $1+3$ DO medical program

Methods: US-based instruction was integrated within the OPP courses for years 1 and 2 of the osteopathic medical education curricula, with emphasis on identification of relevant 
musculoskeletal structures, to augment the findings of the osteopathic physical examination. Based on the OPP course schedule, US modules pertinent to the corresponding musculoskeletal joint anatomy were implemented, whereby, after brief instruction, students practiced peer-to-peer scanning under preceptor supervision in a small group setting at each US station.

Results: Over at least 8 sessions during the 2016-2017 academic year, select pre- to postinstruction evaluation of US concepts understanding as well as formal quantitative student feedback indicated that teaching material was well received by course directors as well as students. Use of US instruction early in the curriculum is perceived by students to be a firsthand means to dynamically visualize key anatomical structures and enhance usefulness of the physical examination.

Conclusion: US as a clinically-applicable technology provides a new active-learning paradigm in osteopathic medical education. Future directions of development of this initiative at the present institution aim to integrate US instruction in anatomy, physiology, and medical skills curriculum.

\section{Limited Number of Faculty for} Throughput? Use Technology to Your Advantage!

Brian Mann; Steven Halm

Campbell University-Jerry M. Wallace School of Osteopathic Medicine

Context: Given the complexity of health systems in the United States, undergraduate medical education must change to better serve the needs of the patient population. Attempts to improve undergraduate medical education are underway to match some of the changes in society, leaving the traditional classroom lecture, and increasing the amount of time in interactive cases.
Objective: Moving toward a more interactive experiential learning environment requires the right ratio of faculty to medical students. Depending on the experiential activity, technology may be able to assist in improving throughput while adding to the educational value and limiting the number of faculty required.

Methods: Assessment tools were embedded into technology platforms, providing real-time feedback to end users. Embedding assessment tools into current technology can be very difficult. The authors offered 2 approaches:

1. Using a live, interactive assessment of students' knowledge can be very effective. This was accomplished through live facilitators working with small groups of no more than 5 students. Each group completed the same interactive activity on a mobile whiteboard device. After each group completed the learning activity, the facilitator provided reflection, critiques, and suggestions for improvement. Using a live assessment tool with technology allowed the facilitator to guide the learners' thought process rather than simply providing answers.

2. Embedded assessments are often part of the technology package provided with software or app design (purchased directly from the company or through a third-party company). Some technology platforms allow for immediate student feedback either in written or verbal form. One strong feature of such a platform allows the administrator or course director to directly observe (see and/or hear) the students' progress. Over months, this information can identify gaps in knowledge and skills, or a lack of progress related to patient-hand-off on clinical rotations. This signal allows for intervention as well as identifying potential areas for remediation. 
Specific products used included the I-PASS Virtual Immersive Learning tool from I-PASS Patient Safety Institute, as well as BaiBoard 3.

Results: One example of assessment that is embedded into the technology platform is found in the I-PASS Virtual Immersive Learning program. The learner can see their written patient handoff while listening to their own patient handoff and synthesis. In a single, easy-to-review dashboard, a faculty member or patient safety officer can review all information documented and listen to recordings of each student patient hand-off and synthesis, allowing for immediate identification of students or residents in need of further training in the patient handoff process.

Conclusion: This type of educational programming bridged undergraduate medical education with graduate medical education, especially related to the patient handoff. Technology may also provide an opportunity to fulfill an entrustable professional activity requirement, such as implementation of a simulated electronic health record. The use of different technologies to enhance the learning environment for undergraduate and graduate medical education can result in more effective use of faculty.

\section{Training Medical Students in Utilization of Humanistic and} Biomechanical Domain Competency Ali Moradi Georgia Campus-Philadelphia College of Osteopathic Medicine

Context: The ability to take a humanistic approach with patients has implications for the physician's own practice as well as for patient outcomes.

Objective: During a routine analysis of student competencies in primary care skills course, it became apparent the students were increasingly challenged with the humanistic approach during student-patient communication skills, both from a confidence and a process point of view. This issue was addressed through review of the course design and the incorporation of intentional progressive development of the humanistic domain. This new approach provided opportunity for medical students to incorporate application of humanism in history taking and physical examination. Student humanistic and biomechanical skills are individually performed with a standardized patient in a timely and safe manner and were analyzed by summative and formative assessment.

Methods: The course director designed a method to incorporate the humanistic domain along with biomechanical skills for every patient encounter scenario. Emphasis on communication skills are strongly implemented throughout history taking and physical examination. Measures such as preserving patient's modesty, providing a compassionate environment, and safety on performance are assessed and evaluated for each student encounter. Formative assessments were used to increase and improve student's performance.

Results: A comparative statistical method was used to characterize 2 different outcomes: before and after change in primary care skills course. Implementation of the humanistic and biomechanical approach resulted in improved aggregate cohort performance proficiency and resulted in increased national passing rate of the COMLEX-USA Level 2-PE.

Conclusion: Students benefited from the experience of being exposed to patient encounters by use of skills learned for incorporation of humanistic and biomechanical domain through patient encounter activity. Features of this method may help struggling curricula or programs to train students in use of both the humanistic and 
biomechanical approaches in primary care skills learning environment.

\section{Career Planning for Osteopathic Medical Schools: A New Approach in Arkansas \\ Holly Profritt \\ New York Institute of Technology College of Osteopathic Medicine at Arkansas State University (NYITCOM at A-State)}

Context: In osteopathic undergraduate medical education there is very little structure or direction in career development curriculum. The Commission on Osteopathic College Accreditation (COCA) stated, "The COM, and/or its parent institution, must provide services devoted career counseling." Compared with medical schools accredited by the Liaison Committee on Medical Education standards, career advising under the COCA is vague in the requirements of institutions to prepare students for the looming and inevitable single graduate medical education accreditation system residency match of 2020. In contrast, schools that are a part of the American Association of Medical Colleges are required to maintain an effective career advising system in place that integrates the efforts of faculty members, clerkship directors, and student affairs staff to assist medical students in choosing elective courses, evaluating career options, and applying to residency programs. These differences in standards between accreditation bodies leave it up to colleges of osteopathic medicine (COMs) to devise career programming with little direction beyond a necessary presence and position. This standard makes it apparent that COMs are falling short in comparison with our allopathic counterparts in the expectation of career development and advising.

Objective: The objective of the career and professional development programming at NYITCOM at A-State is to create a dynamic career development curriculum that will allow students to fully understand their own career development while in time making competitive applicants for graduate medical education programs.

Methods: At NYITCOM at A-State, we are committed to career and professional development of our medical students for all 250 first- and secondyear students. All students at the Jonesboro campus participate in mandatory career development programming throughout their undergraduate medical education. This program was created with measurable learning and objectives and in partnership with the Departments of Academic Affairs and Clinical Education. Programming begins in the first year with students exploring personality types and physician and personal values. The second-year syllabus involves physician workforce issues, researching residency programs and finding the right fit for student interests. The programming incorporates physicians from the community and full-time faculty to show the diversity of specialties. The career development programming does not end in the third year as students leave campus. The integration of technology allows for the students to have access to career development and advising content even in clerkships.

Results: Students at NYITCOM at A-State have demonstrated growth in career development and understanding of their selves and their choices in specialty selection. There is no statistical data yet regarding how this programming will affect match rates for students based on their participation in the program. Limitations to this programming currently are lack of data on match success because the NYITCOM at A-State Inaugural Class will not graduate until 2020. However, responses to survey data show students are gaining exposure to and knowledge of topics not previously included in the current medical school curriculum. 
Conclusion: A structured and measurable approach is needed to ensure osteopathic students are prepared for a more competitive residency match in 2020.

\section{Got Joy? An Osteopathic Approach to The Art of Happiness and Emotional Resilience for Physicians \\ Miko Rose; Jed Magen; Alyse Ley \\ Michigan State University College of Osteopathic \\ Medicine (MSUCOM)}

Context: Suicide is the only cause of death with risks greater for physicians than the general population.

Objectives: This workshop will outline the challenges medical trainees and physicians currently face regarding mental health. The workshop will describe the MSUCOM osteopathic, strengthsbased, happiness-focused intervention model and the unique role it played in promoting healthy behaviors and moderating symptoms of burnout in medical students at 2 medical schools. Presenters will also describe the challenges and evolution of this project, from pilot study to incorporation into medical school curriculum.

Methods: Resident physicians created and taught 60-minute weekly classes for 10 weeks to osteopathic medical students at MSUCOM. Half of each class was devoted to mindfulness training, and the other half of each session was devoted to positive psychiatry/cognitive behavioral therapy interventions. The Beck Anxiety Inventory, the Fordyce Happiness Scale, and the Authentic Happiness Inventory were used to assess the impact of the intervention. Surveys were administered at the onset, midpoint, and termination of the 10-week intervention. Control data were collected from medical students who did not participate in the class sessions.
Results: The mean (SD) Beck Anxiety Inventory scores of participating (intervention) students declined from at the first session to after the last session $13.8(8.1)$ to $6.8(6.8)(P=.007 ; 95 \% \mathrm{CI}$, $-8.089,-1.711 ; \mathrm{df}=9 ; \mathrm{SD}=4.4)$. The mean (SD) Authentic Happiness Inventory Scores of participating (intervention) students improved, increasing from 79.2 (9.6) to 87.3 (13.9) $(P=.046 ; 95 \%$ CI, 0.186, 16.214; df=9; $\mathrm{SD}=11.2)$. After the 10 -week intervention, the mean (SD) Authentic Happiness Scale Score of the intervention group was 87 (11.2) compared with 75 (12.3) in the control group (ANOVA, 8.8; df=1;P=.004).

Discussion: Interactive exercises from these training modules will provide participants direct experience of the material, and facilitated group discussion will lend guidance on implementation strategies participants can take back to their own institutions. Presenters will then lead an interactive group discussion to identify and outline the greatest challenges within individual training institutionsusing this forum for participants to share interventions and ideas among institutions. Ethical approval was granted for this study by the Michigan State University Biomedical and Health Institutional Review Board; approved 1/19/2012, IRB\# 11-1188.

\section{Creating a Research Infrastructure With a Service-Oriented Mindset} L.J. Rush; D.E. Berryman Ohio University Heritage College of Osteopathic Medicine (OU-HCOM)

Context: Collaborative research is a strategic priority at OU-HCOM and complements the elevated status of research in accreditation standards.

Objectives: With campuses in 3 cities and multiple health care partners to consider, creating an infrastructure that fosters collaborative research and serves basic, translational, clinical, and health 
delivery systems researchers is no small task. Research support should decrease administrative burden on faculty while facilitating high-quality research and scholarly activity.

Methods: The first steps toward achieving OU-HCOM's research goals were to determine the infrastructure needs and prioritize areas of strength to maximize the return on investment.

Results: OU-HCOM and its research administration office have adopted a faculty-centered, service-oriented approach to enhancing the existing infrastructure and designing new systems. The presenters enumerated successes and challenges encountered during the creation of a human subjects research unit, establishment of shared equipment core facilities, formation of primary care research support, expansion of molecular and animal-based support, and navigation of collaborations within and external to the university. Attracting and retaining research-oriented faculty who can obtain external funding is critical for sustainability of this infrastructure.

Conclusion: An effective research infrastructure is both a prerequisite for, and a product of, an effective collaborative research program.

\section{From Calamity to Competence: A Joint Interdisciplinary Interprofessional Hand Hygiene (H/H) Teaching Model}

George J. Scott; Pamela Basehore; Cindy Hou Rowan University School of Osteopathic Medicine (RowanSOM)

Context: Clean hands are the single most important factor in preventing the spread of pathogens and antibiotic resistance in the health care setting. The Centers for Disease Control and Prevention (CDC) estimates that nearly 2 million patients in the United States per year get an infection in hospitals, and about 90,000 of these patients die because of their infection. Hand hygiene reduces the incidence of health care-associated infections. RowanSOM developed a joint hospital-COM interdisciplinary program introducing students to infection control and the impact of hand hygiene on patient safety.

Objective: To improve performance of hand hygiene among third- and fourth-year osteopathic medical students (OMS III and OMS IV) on clerkships at a partnering health system.

Design: A cohort study where infection control concepts and best practices for hand hygiene with a laboratory practical were provided annually to all medical students through an interdisciplinary hospital-COM teaching model where student performance of hand hygiene at the hospital was covertly monitored by hospital infection control personnel. Setting: An incident occurred where state department of health inspector observed students fail to perform hand hygiene upon entering/ exiting a patient's room. The inspector reported findings to hospital leaders who prior to the event initiated covert hand hygiene surveillance of all hospital personnel. Hospital statistics revealed students among lowest hand hygiene performers. Participants: All OMS III and OMS IV having previously received hand hygiene training prior to starting clerkships. Main Outcome Measure: Covert determination of student performance of hand hygiene by hospital infection control personnel for the year before and 3 years after initiation of training.

Results: For the year before initiating training, a total of 292 students were monitored covertly by hospital infection control staff, and 198 of these students were observed performing hand hygiene before and after entering a patient's 
room. There were 94 occasions where this behavior was not observed, resulting in a total compliance rate of $68 \%$. For the 3 years following initiation of training, there were 1374 students monitored covertly by hospital staff and 1166 of these students were observed performing hand hygiene before and after entering a patient's room. There were 208 occasions where this behavior was not observed resulting in a total compliance rate of $84 \%$.

Conclusion: Data confirm that mastery has not yet been achieved, but participants have achieved competence. Further reinforcement is necessary, including improved faculty role modeling and identifying teaching opportunities during clinical rounds. National data suggest that hand hygiene performance among health care workers is low for a host of reasons. An educational program that reviews infection control concepts, conveys the importance of hand hygiene, and instructs students in best practices established by national and international organizations will improve hand hygiene compliance among medical students during clerkships. Hand hygiene is important for patient safety, student safety, and compliance with accrediting bodies. The Joint Commission announced beginning January 1, 2018, they will begin monitoring hand hygiene performance of all health care workers engaged in direct patient care during inspections and site hospitals for noncompliance.

\section{Sources:}

https://www.cdc.gov/handhygiene/providers/guideline.html; http:// www.who.int/gpsc/5may/tools/9789241597906/en/; https://www.joint commission.org/topics/hai_hand_hygiene.aspx

\section{A Cost Analysis of Accreditation Reporting \\ Elizabeth Smith-Trigg; Italo Subbarao William Carey University College of Osteopathic Medicine (WCUCOM)}

Context: WCUCOM undertook a cost analysis of accreditation reporting over the course of the 2016-2017 academic year. A centralized, cloudbased database (ProgressIQ) was introduced to aid in data collection for reporting. WCUCOM is a private osteopathic medical school in Hattiesburg, Mississippi, with a class of 100 . Since its inception in 2010, all departments took on individual roles of providing data for accreditation reports, which has become time consuming and costly.

Objective: The WCUCOM Assessment Office undertook an initiative to find a centralized cloudbased solution for effective data management and accreditation reporting. A pre- and post-cost analysis was conducted to evaluate the effectiveness of the new system compared with the old system.

Methods: All areas of data management and warehousing at the COM were integrated into a centralized data management program. The areas included student affairs (American Association of Colleges of Osteopathic Medicine [AACOM] report), preclinical (CANVAS), clinical rotations (EValue), and assessment (National Board of Osteopathic Medical Examiners). ProgressIQ, a cloud-based, centralized database with permissions was utilized as the central repository for the data. With the purchase of the new system, WCUCOM Assessment Office undertook a cost analysis of 3 accreditation reports to determine the utility of ProgressIQ. ProgressIQ included a 1-time startup cost of approximately $\$ 60,000$ based on calculations of software and administrative transfer of all WCUCOM data from the warehouse. 
Results: Throughout the 2016-2017 academic year, WCUCOM conducted a cost analysis of 3 accreditation reports (Commission on Osteopathic College Accreditation, AACOM, Southern Association of Colleges and Schools). With the use of the ProgressIQ, WCUCOM saved approximately $\$ 10,000$ per report, an annual savings of approximately $\$ 30,000$.

Conclusion: The break-even point for this investment is projected at 2 years. At the same time, the system allows for additional features such as real-time data monitoring and student status tracking. Outcomes support a strong recommendation for moving to a cloud-based, centralized database.

\section{Implicit Bias: Where It Comes From and What It Looks Like \\ Linda Grace Solis \\ University of the Incarnate Word School of \\ Osteopathic Medicine}

Context: "If we are human, we are biased." This statement causes a strong reaction in many people. Good people do not want to describe themselves as biased; the assumption is that "bias" equals "racism." In the case of implicit bias, this may or may not be true. Certainly, those labeled as "racist" hold bias-explicit, outward bias. Others also hold bias, but it is implicit, or unconscious.

Objective: To understand that bias is formed as identity is formed-by family, society, and environment.

Data: Research has not provided a concrete method to eradicate implicit bias altogether, but there are research-based tools available that uncover specific implicit biases, along with proven techniques to ensure that biases do not negatively impact one's colleagues, patients, and others with whom an individual may come in contact.
Results: Individual reflection and group discussion resulted in a toolkit for uncovering and mitigating our implicit bias. Implicit association tests (IATs) were discussed as a way to discover our individual implicit biases. IATs can be found on the Harvard Implicit website. Ways to increase self-awareness, such as keeping a journal, developing the habit of mindfulness, and asking for feedback, were also discussed as tools to help mitigate implicit bias. Since "fast-brain" thinking is one of the causes of implicit bias, the group talked about ways to slow thinking such as using breathing techniques to create a space between stimulus and response. The group was also provided with a mnemonic (LIVE) to help them use micro-affirmations to make sure the people around them feel listened to, included, valued, and engaged. Finally, reflection questions were provided as a framework for first steps into intentional mindfulness and self-awareness

Conclusion: It is important for osteopathic medical students and educators, as leaders in medicine with a focus on mind, body, and spirit, to understand implicit bias, to reflect on their own biases and to learn techniques to minimize its harmful effects.

\section{Using Technology to Stay Up Close and Personal With Students at Distant Clerkship Sites}

Lisa Streyffeler; Rebecca D. Shaw; Tyler Moore; Matthew Drilling; Leslie Wimsatt

Des Moines University College of Osteopathic

Medicine (DMU-COM)

Context: Web-based, asynchronous learning modules can be used to provide uniform learning opportunities at distant locations, but little is known about the potential for using other types of enabling technologies in the delivery of real-time instructional experiences to clerkship students.

Objective: To determine the feasibility and efficacy of using weekly, online didactic sessions to 
support clerkship student education, Skype for Business was piloted for use within 2 required OMS III clerkships (psychiatry and obstetrics and gynecology [OB/GYN]).

Methods: Questionnaires were distributed during 2 clerkship rotations to determine outcomes related to piloting weekly, real-time didactic experiences using Skype for Business with thirdyear DMU-COM students enrolled in psychiatry and $\mathrm{OB} / \mathrm{GYN}$ at distant rotation sites. Ten groups of 8 to 18 students for each specialty participated over the course of 4 required, month-long clerkships ( $\mathrm{n}=26$ Psychiatry; $\mathrm{n}=50$ OB/GYN). In psychiatry, students were given time to reflect on the experience of encountering significant human suffering, as well as giving oral case presentations with associated miniature didactic presentations. In $\mathrm{OB} / \mathrm{GYN}$, Articulate Storyline software allowed for more complex activities beyond group discussion using a multistep, interactive case scenario along with individual student oral presentations. Assessment of this learning activity ranged from student participation rates and user satisfaction to IT effectiveness. The psychiatry questionnaire contained 5 open-ended questions regarding the student experience, whereas OB/GYN solicited first/last class day responses to 16 structured questions focused on student confidence with various professional skills and student level of connection with the host campus faculty.

Results: Student questionnaire recipients responded to the in-class questionnaires (response rate $75 \%-100 \%$; $=76$ ). In psychiatry, 25 students reported finding the sessions useful (96\%) and preferable to asynchronous online didactic modules (95\%) while 11 (42\%) appreciated learning from the shared experiences of colleagues in the online environment. In OB/GYN, the before and after percentage of students reporting a connection to faculty increased from $27 \%$ to $69 \%$ (from 14 to 35 students) while students reporting confidence delivering bad news increased from $17 \%$ to $31 \%$ (from 9 to 16 students) and those reporting confidence with case presentations increased from $34 \%$ to $73 \%$ (from 17 to 37 students). The percentage reporting confidence selecting appropriate laboratory tests/imaging increased from $29 \%$ (15) to $60 \%$ (30) students. Additional results indicate significant before/after gains in student confidence locating scientific evidence to support diagnostic reasoning and treatment planning $(P=.02)$. Faculty interviews revealed that the experience provided a unique and much-appreciated opportunity for interdisciplinary and interdepartmental collaboration.

Conclusion: Although limited to a small pilot study at a single osteopathic medical school, the results of this experience suggest the feasibility and efficacy of developing and implementing an educationally useful, technically manageable didactic session for online, synchronous delivery to students at distributed clerkship locations.

\section{Interprofessional Collaboration Between Graduate Medical Education and Health Science Programs \\ Suporn Sukpraprut-Braaten; Debbie Waggoner; Stacy Zimmerman \\ Kansas City University of Medicine and Biosciences}

Context: Interprofessional education is a required experience in many medical education programs. Development of an effective interprofessional collaboration has the potential to enhance training and patient care and improve training programs.

Objective: To create an effective model incorporates residents, medical students, pharmacy residents, and other medical professions to provide optimal patient care. An effective model 
incorporates residents, medical students, pharmacy residents, and other medical professions to provide optimal patient care and enhance interpersonal communication skills and elevate the cognitive level of learning.

Methods: The objectives of the intervention were to (1) meet the Accreditation Council for Graduate Medical Education (ACGME) requirements and Milestones related to interprofessional collaboration, communication, and education; (2) integrate ACGME scholarly activity requirements with interprofessional involvement and collaboration with an IRB committee liaison; (3) design a plan to assist the graduate medical education (GME) department, a GME consortium, and local university programs to meet accreditation standards and enhance education; (4) expound on the practical implementation of the interprofessional collaborative plan; (5) formulate a crossover research curriculum to enhance the students' and residents' knowledge in evidence-based medicine and principles of clinical research; (6) implement standardized evaluation strategies for ongoing educational and research program improvement; and (7) design a framework to enhance the residents' and students' knowledge of the principles of interprofessional dynamics and education.

Results: There were 7 publications, 15 podium presentations, 23 research posters, and 15 case report posters since 2016 as the result of the interprofessional collaboration between the GME and health science programs.

Conclusion: The interprofessional education model provided opportunities for students and residents from multiple disciplines to collaborate on research projects and quality improvement and gain teaching and learning opportunities. The collaboration encouraged them to work as an interprofessional team for patient care.

\section{Collaboration Opportunities With} Electronic Learning Platforms

Sean Tackett ${ }^{1}$; Mark R. Speicher ${ }^{2}$; Christina Goode ${ }^{3}$; Grace L. Cisek ${ }^{4}$

${ }^{1}$ Johns Hopkins Bayview Medical Center;

${ }^{2}$ American Association of Colleges of Osteopathic

Medicine; ${ }^{3}$ Western University of Health Sciences

College of Osteopathic Medicine of the Pacific;

${ }^{4}$ Midwestern University Arizona College of

Osteopathic Medicine

Context: Today's medical students have many commercial study aid resources to choose from and are increasingly using them, with mixed results for both coursework and high-stakes standardized testing. Faculty are often unfamiliar with these resources, and failure to acknowledge and integrate students' use of them into their teaching can create a disconnect for students and faculty, making learning more difficult and teaching less rewarding. Osteopathic medical faculty, in particular, are under greater pressure to ensure their graduates are competitive for residency. Integrating commercial study resources offers an opportunity for faculty and colleges of osteopathic medicine to explore the learning methods of their students, especially struggling students.

Objective: To assess the extent of integration of a commercial study tool in 2 of its partner institutions.

Methods: We used data from Osmosis, a multimedia, multimethod study program featuring a question bank, flashcards, video lectures, and other materials. Data on student use of osmosis was made available to both a master's program for premedical students at a large western medical school, and a second-year board review elective at a large southwestern medical school. The data in the premedical program were integrated into several courses to ensure learning of the study tool. In the OMS II year, the elective program encouraged individual faculty to integrate the Osmosis materials into their sections. 
Results: In the premedical program, students largely accepted the Osmosis resources, though there was a great variation in utilization. However, in the first year of medical school (the first year after the program), only $50 \%$ of the students continued using Osmosis. In the OMS II elective, only 2 faculty members, the pathology and psychiatry faculty, integrated Osmosis into their programs, but there was high use of the program among students in these areas.

Conclusion: Students accepted the Osmosis tools initially, but not all students were able to integrate the tools into their study plans long term. Faculty who integrated Osmosis had success doing so in both programs, but other faculty showed some resistance to integrating the tool as well. More research is needed to determine reason for student and faculty resistance to integrating Osmosis into their study plans.

\section{Mentoring and Advising Osteopathic Medical Students in the First 2 Years: Through Anatomy and Beyond N.T. Zaveri'; M.E. Coty ${ }^{2}$; F. Liuzzi ${ }^{2}$ \\ ${ }^{1}$ Touro University Nevada College of Osteopathic Medicine; ${ }^{2}$ Lake Erie College of Osteopathic Medicine-Bradenton}

Context: This study investigates the effects of early intervention and mentorship during anatomy, on outcomes in preclinical osteopathic medical education.

Objectives: To determine if a structured mentoring program, when provided early, results in improved outcomes during the anatomy course. An important secondary objective is to determine if mentorship influenced longitudinal outcomes and/or board scores.

Methods: During orientation, students are assigned to a faculty advisor, and they are required to meet monthly during years 1 and 2 . Current curriculum is entirely problem-based learning (PBL) during the first 2 years of medical education, in which all basic medical sciences are learned devoid of lectures, with the exception of anatomy. Anatomy is delivered in the first 11 weeks of the first semester prior to beginning PBL. After the first 2 weeks, a quiz is given and students in academic difficulty are identified. An average number of 20 to 35 students fail the first anatomy quiz. At this point, a structured mentoring program, in which faculty and administrators intervene, is described. When students fail the first anatomy quiz, faculty advisors are notified. Students then receive 1-on-1 counseling sessions with their advisors, experienced anatomy faculty, and the preclinical dean to provide mentorship. Student performance data on subsequent anatomy examinations is tracked and the intervention (mentorship) process is repeated. This study design included 200 students in each class of first-year osteopathic medical students. Outcomes on the quiz and final course for anatomy, as well as Comprehensive Osteopathic Medical Licensing Examination-USA (COMLEX- USA) Level 1 scores, were gathered for each student.

Following anatomy, a similar process of early identification and intervention is utilized for PBL and all other coursework over the first 2 academic years. Longitudinal data shows improvement on COMLEX-USA Level 1 following early intervention. The main outcome measures in this study are pass rates for the anatomy quiz and the overall anatomy course, and COMLEX-USA Level 1. Presented are data for 3 classes for the 2 former examinations, and data for 1 class for the latter (COMLEX-USA Level 1).

Results: The data show that the pass rate for the anatomy course for the group that received the intervention is very similar to the group that passed the quiz and did not need intervention, for all classes shown. Longitudinal data with COMLEX-USA 
Level 1 scores shows that a $100 \%$ of students who received early intervention (total 27 from the class of 2019) passed the board examination, while 2 students who did not receive early intervention failed the board examination, resulting in a $98.7 \%$ pass rate.

Conclusion: Outcomes show that structured early mentorship is a very effective tool to help students succeed in their preclinical education. One limita- tion of this study is that data have not been provided to correlate outcomes from anatomy to other foundational basic sciences in PBL to COMLEX-USA Level 1. Additional data and research are needed to assess the effect of early mentorship on success in other coursework and other outcomes such as the United States Medical Licensing Examination Step 1.

๑) 2018 American Osteopathic Association 